\title{
Nobel award stirs up debate on nitric oxide breakthrough
}

[LONDON] The Nobel committee has once again sparked controversy, particularly in Britain, with the award this week of the 1998 prize for physiology or medicine to pharmacologists Robert Furchgott, of the State University of New York, Louis Ignarro, of the University of California, Los Angeles, and Ferid Murad, of University of Texas Medical School in Houston.

No-one is disputing that the pioneering work of the three US researchers on nitric oxide as a signalling molecule in the cardiovascular system (see box) deserves the recognition of the committee. But many feel that a fourth name should also have been recognized - that of Salvador Moncada, currently director of the Wolfson Institute for Biomedical Research at University College London.

The controversy originates in 1987, when a paper by Ignarro confirming the identity of endothelium-derived relaxing factor (EDRF) and nitric oxide, as had been hypothesized separately by him and Furchgott, appeared in the Proceedings of the National Academy of Sciences (84, 9265-9269; 1987).

The publication came six months after a similar report in Nature by Moncada and colleagues, working in London, who had already concluded that EDRF and nitric oxide were identical (see Nature 327, 524-526; 1987). Writing in the same issue of Nature, Paul Vanhoutte, then at the Mayo Clinic in Rochester, Minnesota, acclaimed the finding as "the climax of one of the most exciting sagas in vascular physiology and
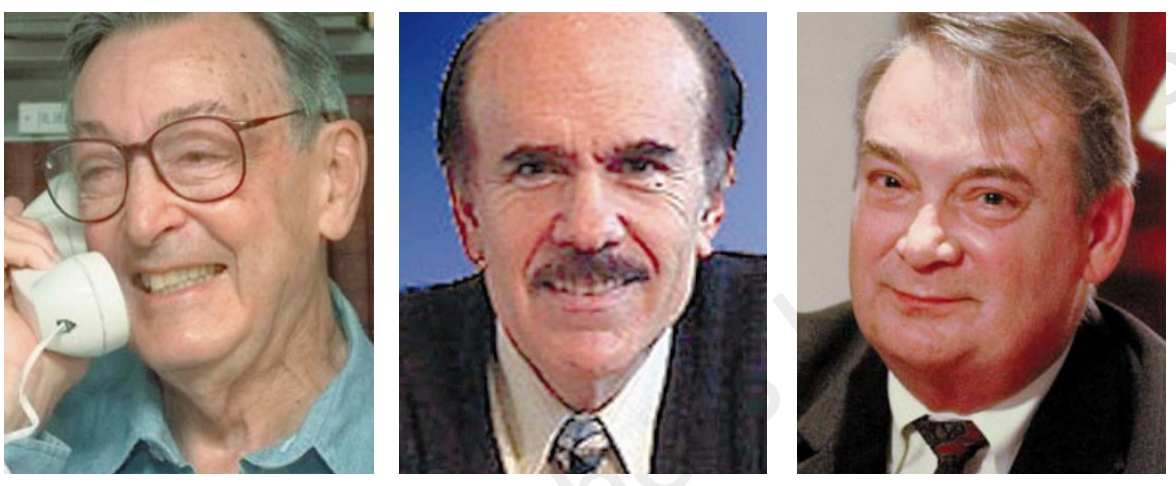

The winners: Robert Furchgott (left), Louis Ignarro and Ferid Murad.

pharmacology". Moncada's achievements were recognized when he was elected as a foreign associate of the National Academy of Sciences in 1994. The citation says that he "discovered that mammalian vascular tissues generate nitric oxide that is biosynthesized from L-arginine. He elucidated the relevance of this pathway as a universal transduction mechanism for the regulation of cell function and communication".

Moncada expressed surprise on Monday (12 October) at the decision of the Nobel committee to award the medicine prize to Furchgott, Murad and Ignarro. His disappointment was shared by Sir John Vane, joint winner of the 1982 Nobel prize for his work on prostaglandins.

Although delighted that the committee has recognized the importance of the nitric oxide field, Vane expressed regret that Moncada's contributions were not acknowl-

\section{Hidden role of a gas of many parts}

[LONDON] Interest in nitric oxide has exploded in recent years due to the recognition that the gas plays an important role in many physiological processes, and that manipulating the nitric oxide signalling pathway can have major medical benefits. But, only 30 years ago, the idea that a gas could have biological functions would have seemed far-fetched.

In 1980, Robert Furchgott reported that acetylcholine could contract or relax the smooth muscle surrounding blood vessels, but that this relaxation only occurred if the endothelium - the lining of the blood vessel - was intact (see Nature 288, 373-376; 1980). The implication was that a signal molecule was being released by endothelial cells and acting on the muscle cells, and Furchgott called the postulated molecule endothelium-derived relaxing factor - EDRF.

The link with nitric oxide was not immediately obvious. EDRF was highly labile, and candidate molecules, such as prostacyclin and other prostanoids, were soon ruled out. But in the 1970s Ferid Murad had discovered that the gas could activate guanylate cyclase, the enzyme that mediates the production of the signalling molecule cyclic GMP.

Murad also showed that nitroglycerin and other vasodilating drugs released nitric oxide. But the physiological relevance of this discovery remained unknown. The various links in the story were only pulled together at a conference in 1986 when Furchgott and Louis Ignarro, who had been working independently of each other, hypothesized that EDRF and nitric oxide were identical. edged. But he believes that the prize is thoroughly deserved by Furchgott — without whom "the field would not even exist" and says that he had nominated both Furchgott and Ignarro for the prize.

More outspoken support for Moncada comes from Nobel laureate Cesar Milstein of

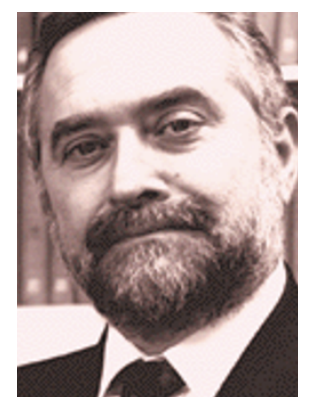

Moncada: disappointed at lack of recognition. the MRC Laboratory of Molecular Biology in Cambridge. Milstein won the 1984 Nobel prize for his work on monoclonal antibodies, and dug deep into the nitric oxide literature following the award in 1996 of the prestigious Albert Lasker Medical Research Award to Furchgott and Murad.

Milstein argues on the basis of his investigations that Moncada was the first to take seriously the suggestion by Furchgott and Ignarro that EDRF might be identical with nitric oxide - and did the key experiments that made the earlier hypothesis physiologically meaningful.

Characterizing the decision as "scandalous", Milstein argues that the committee made a serious blunder by excluding Moncada from the awards.

Milstein says he does not believe that the omission of Moncada will seriously damage British research. But he regrets that this research will not get the fillip it deserves, and admits that the omission leaves him "uneasy about the way that recognition is being given at the level of major prizes, and in terms of careers and publications".

Not all researchers are as critical. Jonathan Stamler, of Duke University Medical Center in North Carolina, who also works on nitric oxide in the cardiovascular 
system, says he is very pleased that the work of Furchgott, Ignarro and Murad has been recognized.

Stamler accepts that Moncada "had the vision to appreciate the field in the broader sense and to shoulder the burden of later research". But he believes that earlier work had already opened the way for the later discoveries, as the Nobel committee has recognized.

Solomon Snyder of Johns Hopkins University in Baltimore, Maryland, says he agrees with this interpretation, and accepts that the committee would have done much scholarly work before making its decision.

$\mathrm{He}$ says the medical significance of Murad's work on nitroglycerin is enormous. Murad showed that the active metabolite of nitroglycerin is nitric oxide, leading drug companies to search for other drugs that could release the gas.

Ironically, Alfred Nobel, the inventor of nitroglycerin is kept in check by a porous material composed of diatoms — was ordered by his doctor to "eat" nitroglycerin for a heart condition, but refused to take it.

Nitroglycerin is a potent drug now used in the treatment of angina. Just $0.5 \mathrm{mg}$ placed under the tongue can make a patient suffering an angina attack feel better within minutes, due to the dilation of the affected blood vessels.

It is now clear that nitric oxide is the major determinant of blood pressure. But its medical significance does not stop there. In 1992 Snyder and colleagues showed that nitric oxide synthase (NOS), the enzyme that produces nitric oxide, is expressed in neurons in the penis and that nitric oxide mediates erectile function.

RoryHowlett dynamite - in which the explosiveness of

\section{Physicists rewarded for 'fractional electrons'}
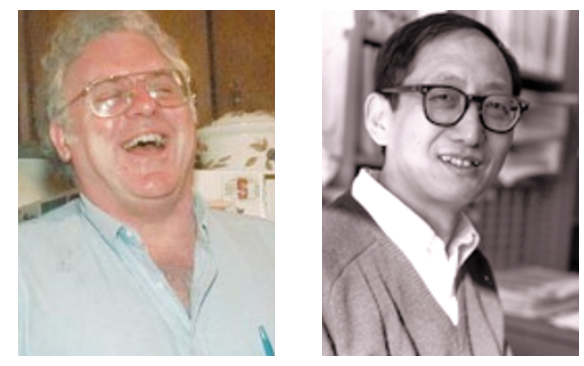

[LONDON] This year's Nobel prize for physics has been awarded to the researchers who first observed and explained the fractional quantum Hall effect. This is the effect in which an electric current within a two-dimensional conducting material appears to be made up of charge carriers bearing a fraction of the charge on an electron.

Horst Störmer of Columbia University, New York, and Bell Laboratories, and Daniel Tsui of Princeton University, who both saw the effect experimentally in 1982, share the prize with Robert Laughlin of Stanford University, California, who provided a theoretical explanation shortly afterwards.

The standard Hall effect is the lateral deflection of moving charge carriers - an electric current - in a magnetic field. It was discovered in 1879 by Edwin Hall, and today provides the basis for determining the charge and density of charge carriers in a semiconductor (electrons and holes are deflected in different directions).

\section{Theoretical chemistry makes its mark}

[LONDON] The award of the Nobel prize for chemistry to Walter Kohn of the University of California at Santa Barbara and John Pople of Northwestern University, Illinois, chemistry is now a tool at the chemist's disposal to equal any experimental or analytical technique.

Kohn provided the theoretical framework for calculating the electronic structure of molecules without having to grapple with the formidable task of solving the full Schrödinger equation. He showed that the total energy of a system can be expressed simply in terms of the distribution of its electron density, without regard to the details of the electron motions; coordinates, and the energy is a 'function of a function', or a functional.

Kohn developed what was later to be called density functional theory in 1964, with applications in physics in mind. This theory is now widely used to calculate the signals a recognition that computational the density is a function of the spatial

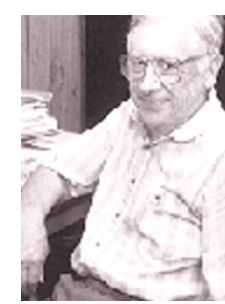

Pople: work has 'led to an industry'. for calculating the electronic structure of molecules and the nature of their interactions and reactions.

Current modifications of this program are now used by thousands of chemists throughout the world, according to Roberto Car of the Ecole Polytechnique Fédérale in Lausanne, Switzerland. "[Pople] has built an industry around this," says Car.

That view is echoed by Nicholas Handy at the University of Cambridge, who feels that the laureates are "exactly the right two people" to be recognized.

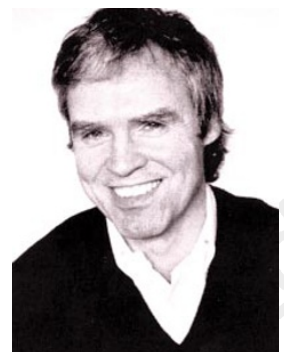

Physics laureates: Robert Laughlin (left) shortly after hearing of his award, Daniel Tsui (centre) and Horst Störmer.
In 1980 Klaus von Klitzing found that, when the charge carriers are confined within a very thin conducting film (that is, in two dimensions), the magnitude of the Hall current (or, equivalently, the conductance of the material) no longer varies smoothly with magnetic-field strength at very low temperatures.

Instead, the conductance varies with field strength in a series of abrupt steps. In other words, the conductance is quantized: it changes in integral multiples of the fundamental quantum unit of conductance, $e^{2} / h$ (where $e$ is the charge on the electron and $h$ is Planck's constant).

The fractional quantum Hall effect represents a deeper puzzle, since it seems to reveal a change in the nature of the fundamental particles. Much the same can be said of superconductivity, in which electrons appear to attract one another (or, more properly, to show bosonic instead of fermionic behaviour), and of superfluidity, in which the atoms of the superfluid no longer generate viscosity.

The FQHE was seen by Tsui and Störmer for the transport of a two-dimensional electron 'gas' in a semiconductor heterostructure fabricated by Art Gossard, now at the University of California at Santa Barbara. On applying magnetic fields of up to 30 tesla to a sample cooled to about a tenth of a degree kelvin, they observed jumps in conductance with a value of $e^{2} / 3 h$, implying that the charge carriers had a fractional charge of $e / 3$. Subsequent studies revealed charges of $2 e / 5,3 e / 7$ and other (odd-denominator) fractions.

Laughlin proposed that the magnetic flux lines penetrating the sample encourage the charge carriers to condense into quasiparticles. He demonstrated that such quasiparticles act as though they have fractional charges with the values seen in the experiments.

The crucial insight, says Moty Heiblum of the Weizmann Institute in Israel, was the recognition of the role of electron correlations. In semiconductor physics, says Heiblum, "all of us managed to work with a single-electron picture for many years". But the study of strongly correlated electrons in solid-state physics has now become an important field of research.

PhilipBall 